\title{
Impact of High Intensity Exercise on Muscle Morphology in EAE Rats
}

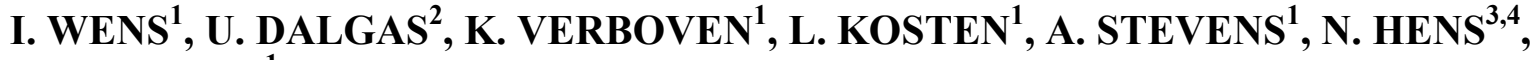 \\ B. O. EIJNDE ${ }^{1}$
}

${ }^{1}$ REVAL Rehabilitation Research Center, BIOMED Biomedical Research Institute, Faculty of Medicine and Life Sciences, Hasselt University, Diepenbeek, Belgium, ${ }^{2}$ Section of Sport Science, Department of Public Health, Aarhus University, Aarhus, Denmark, ${ }^{3}$ Interuniversity Institute for Biostatistics and Statistical Bioinformatics, Hasselt University, Diepenbeek, Belgium, ${ }^{4}$ Center for Health Economics Research and Modelling Infectious Diseases, Vaccine and Infectious Disease Institute, University of Antwerp, Antwerp, Belgium

Received May 5, 2014

Accepted April 10, 2015

On-line June 5, 2015

\section{Summary}

The impact of high-intensity exercise on disease progression and muscle contractile properties in experimental autoimmune encephalomyelitis (EAE) remains unclear. Control (CON) and EAE rats were divided into sedentary and exercise groups. Before onset (experiment $1, \mathrm{n}=40$ ) and after hindquarter paralysis (experiment 2, n=40), isokinetic foot extensor strength, cross sectional area (CSA) of tibialis anterior (TA), extensor digitorum longus (EDL) and soleus (SOL) and brain-derived neurotrophic factor (BDNF) levels were assessed. EAE reduced muscle fiber CSA of TA, EDL and SOL. In general, exercise was not able to affect CSA, whereas it delayed hindquarter paralysis peak. CON muscle work peaked and declined, while it remained stable in EAE. BDNF-responses were not affected by EAE or exercise. In conclusion, EAE affected CSA-properties of TA, EDL and SOL, which could, partly, explain the absence of peak work during isokinetic muscle performance in EAE-animals. However, exercise was not able to prevent muscle fiber atrophy.

\section{Key words}

EAE - Muscle strength - Muscle fiber characteristics • High intensity treadmill running $\bullet$ BDNF

\section{Corresponding author}

I. Wens, REVAL - Rehabilitation Research Center, Biomedical Research Institute (BIOMED) Hasselt University, Martelarenlaan 42, 3500 Hasselt, Belgium. E-mail: inez.wens@uhasselt.be

\section{Introduction}

Multiple sclerosis (MS) is an inflammatory demyelinating disease of the central nervous system (CNS) with subsequent destruction of myelin, oligodendrocytes and axons in the brain, brain stem and spinal cord (Compston and Coles 2002, Noseworthy et al. 2000). Muscle weakness and muscle fatigue are two frequently occurring symptoms in MS. Consequently, many MS patients show a reduced daily life physical activity level, often leading to inactivity related loss of muscle strength and muscle mass and reduced quality of life (Petajan and White 1999, White and Dressendorfer 2004). Several studies already investigated muscle properties in MS patients (Carroll et al. 2005, De Haan et al. 2000, Garner and Widrick 2003, Kent-Braun et al. 1994, 1997, Ng et al. 2004, Sharma et al. 1995, Wens et al. 2014). The effects of MS per se on muscle fiber characteristics such as cross sectional area (CSA) and fiber proportion has already been evaluated by our group, reporting a reduced muscle strength and a smaller m. vastus lateralis fiber CSA in MS patients compared to healthy controls. Furthermore, a selective type II(a) atrophy was reported in MS patients and muscle fiber CSA was highly correlated with muscle strength, emphasizing the relation between muscle fiber CSA, proportion and muscle strength/performance (Wens et al. 2014). Similar to other pathologies, it is clear that muscle

PHYSIOLOGICAL RESEARCH • ISSN 0862-8408 (print) • ISSN 1802-9973 (online)

(c) 2015 Institute of Physiology v.v.i., Academy of Sciences of the Czech Republic, Prague, Czech Republic

Fax +420 241062 164, e-mail: physres@biomed.cas.cz, www.biomed.cas.cz/physiolres 
weakness in MS can, at least in part, be reversed by physical exercise (Broekmans et al. 2010, 2011, Dalgas et al. 2008, Hortobagyi et al. 2000, Rietberg et al. 2005, Stuifbergen 1997). The impact of physical exercise on muscle fiber characteristics in MS has only been investigated by Dalgas and co-workers, reporting increased $m$. vastus lateralis mean fiber CSA $(8 \pm 15 \%)$ after 12 weeks of progressive resistance training (Dalgas et al. 2010). The impact of other training modalities such as aerobic training on muscle morphology has not been investigated yet. Furthermore, several authors suggested that MS patients could benefit more from higher aerobic training intensities (Collett et al. 2011, Dalgas et al. 2008, 2009a, 2009b). However, it is unclear whether high intensity exercise could be tolerated in MS patients, due to the possible worsening of symptoms. To address this concern and before evaluating the effects of high intensity aerobic training on muscle morphology and function in MS patients, it therefore seems relevant to investigate this first in a MS animal model, experimental autoimmune encephalomyelitis (EAE). In addition, applying the EAE model further allows analysis of whole muscle samples contrary to muscle biopsies only in humans.

The EAE animal model allows investigation of an inflammatory demyelinating disease of the CNS, that is often used to investigate demyelination of the CNS in general, and MS in particular (Constantinescu et al. 2011, Raine and Traugott 1984). Briefly, after an external immunization step (injection of a myelin antigen) to induce the disease, myelin reactive T-cells are activated, crossing the blood brain barrier and causing inflammation after reactivation in the CNS (Ben-Nun and Cohen 1982, Ben-Nun et al. 1981, Vandenbark et al. 1985). This model can be induced in mice (Olitsky and Yager 1949), rats (Lipton and Freund 1953), hamsters (Tal and Behar 1958), marmosets (Genain and Hauser 1996), rabbits (Morrison 1947), goats (Innes 1951), sheep (Innes 1951) and dogs (Thomas et al. 1950) and can, depending on the genetic background of the animal strain and the myelin protein, follow an acute monophasic, a progressive or a more chronic relapsing-remitting disease course. Clinically, exacerbations are characterized by hindquarter paralysis, starting at the tip of the tail and progressing to the trunk, and are scored on a scale ranging from 0 (no signs) to 5 (death) (Polfliet et al. 2002).

Literature investigating the impact of EAE and physical exercise on muscle morphology is sparse and partly conflicting, but most results are promising
(Broekmans et al. 2009, De Haan et al. 2004, Eijnde et al. 2008, Le Page et al. 1994, 1996, Rossi et al. 2009, Wens et al. 2015). De Haan and co-workers demonstrated a significant EAE-induced loss of gastrocnemius muscle mass ( $33 \%$ ), reduced muscle fiber CSA of all fiber types (40-50\%) and reduced maximal muscle force and power (58 and $73 \%$, respectively) (De Haan et al. 2004). We previously reported comparable results for muscle strength and type IIb fiber CSA of $m$. tibialis anterior (TA) and $m$. extensor digitorum longus (EDL) (Wens et al. 2015). In accordance to Le Page and co-workers (1994) we also demonstrated that daily high intensity $(1 \mathrm{~h} /$ day $)$ treadmill exercise, during the inflammatory period (days 1 to 10 after EAE induction), delayed EAE associated hindquarter paralysis onset, while low and moderate exercise intensities had no effect on the disease course (Broekmans et al. 2009, Wens et al. 2015). Furthermore, inactivity, due to EAE induced paralysis, prevented exercise effects on muscle fiber characteristics (Wens et al. 2015).

These findings warrant further exploration of the beneficial disease modifying effects of exercise and the underlying neurological basis of these benefits, since the underlying mechanisms of the therapeutic effects of exercise in MS patients are not clear yet. In this respect, it is important to mention that exercise can affect the production of brain-derived neurotrophic factor (BDNF) (Castellano and White 2008, Cotman and Berchtold 2002), a neurotrophin that often plays an important role in neurogenesis (Rossi et al. 2006) and synaptic plasticity (Cotman and Berchtold 2002, Dalgas and Stenager 2012, Lu 2003, McAllister et al. 1999, Schinder and Poo 2000). In fact, BDNF is an important key-regulation mediator in this process due to its activity depended regulation ( $\mathrm{Lu}$ 2003) and neuroprotective potential (Lewin and Barde 1996). Interestingly, Rojas Vega et al. (2006) showed that $10 \mathrm{~min}$ of moderate aerobic cycling was not sufficient to increase the serum BDNF concentration above the pre-exercise level, whereas a single bout of maximal incremental exercise resulted in a significant increase in serum BDNF. In accordance with these results, Ferris et al. (2007) indicated that the magnitude of the increase in serum BDNF concentration during exercise is dependent on exercise intensity. In MS, Gold and co-workers showed that $30 \mathrm{~min}$ of moderate aerobic exercise significantly induced BDNF production in MS patients to the same extent as in healthy controls (Gold et al. 2003). However, Schulz et al. (2004) and Castellano and White (2008) investigated the effect of 8-weeks of 
aerobic bicycle training of moderate intensity in MS patients and found no changes in basal nor exerciseinduced serum BDNF levels. Moreover, an investigation of the impact of high intensity aerobic exercise on BDNF levels in MS patients is warranted. Since tolerance to high intensity aerobic exercise may be limited in persons with MS, initial studies applying the EAE model seem appropriate.

Consequently, the current study aimed to investigate the impact of high intensity aerobic exercise on disease course, muscle morphology and BDNF release in EAE animals, immediately before (experiment 1) and after (experiment 2) hindquarter paralysis. We hypothesized that high intensity aerobic exercise affects disease course, muscle contractile properties and BDNF release, keeping in mind that paralysis induced inactivity may temper these effects. In doing so, a protocol of regular exercise prior to the start and development of the disease seems as a useful design when investigating the relation between regular physical exercise, motor function and disease management during an acute relapse in EAE (and possibly MS).

\section{Methods}

\section{Animals}

Eighty female Lewis rats (age 6-7 weeks, body weight 100-120 g, Harlan CPB, Zeist, The Netherlands) were individually housed in a conventional facility, provided with enrichment, in the animal facilities at Hasselt University, on a constant light:dark cycle (12 h:12 h), a temperature of $22^{\circ} \mathrm{C}$ and a relative humidity of $22-24 \%$. Rats were fed ad libitum with normal rat pellets (Carfil RN-01-K12, Harlan). The animal Ethics Committee of Hasselt University approved the study protocol in accordance with the national and European legislation. The National Research Council's guide for the care and use of laboratory animals was followed.

\section{Study design}

Following acclimatization and adaptation (day -21 to -15$)$, animals were randomized into two experiments ( $n=40$ per experiment, Fig. 1). On day -14 rats were, in each experiment, divided into two subgroups: a sedentary group (SED, $n=20$ ) and an exercise group (EX, $n=20)$. Due to the nature of the trial, the researchers involved in the daily progression of the project could not be blinded to group allocation. As performed previously (Broekmans et al. 2009, Eijnde et al. 2008, Wens et al. 2015), EX animals were familiarized to treadmill running during the habituation period (day -14 to -1 ) by progressively increasing running duration and intensity, until a running duration of $1 \mathrm{~h}$ and a running speed of $18 \mathrm{~m} / \mathrm{min}\left(25^{\circ}\right.$ inclination) was reached. During this habituation period EX animals were encouraged to run by means of short electrical shocks. These shocks lasted $<1 \mathrm{~s}$ and usually occurred a few times during the training sessions. In order to induce comparable levels of stress, SED animals were seated on the stationary treadmill $(1 \mathrm{~h})$ on a daily basis. From the habituation period onwards, daily food intake and body weight were registered. At day 0, SED and EX groups were subdivided into a healthy control group $\left(\mathrm{CON}^{\mathrm{SED}}, \mathrm{CON}^{\mathrm{EX}}\right)$ and an EAE group $\left(\mathrm{EAE}^{\mathrm{SED}}, \mathrm{EAE}^{\mathrm{EX}}\right)$, followed by EAE induction. Hereafter, EX rats exercised daily for $1 \mathrm{~h} /$ day, during 10 consecutive days, until progressive hindquarter paralysis prevented this. At day 0 and 9 arterial blood samples were collected from the tail and serum samples were stored at $-80{ }^{\circ} \mathrm{C}$.

Experiment 1: At day 10, before onset of hindquarter paralysis, treadmill training was terminated. To avoid acute exercise-induced training effects, tests were performed forty-eight hours after the last exercise bout. First, an additional arterial blood sample from the tail was collected, and all animals were then anaesthetized using an intraperitoneal injection of pentobarbital sodium (5 mg $\left.100 \mathrm{~g}^{-1} \mathrm{BW}\right)$. Following determination of repetitive isokinetic foot extensor performance of the left hind limb (see details later), m. extensor digitorum longus (EDL), m. tibialis anterior (TA) and $m$. soleus (SOL) of the right hind limb were dissected and freed of connective tissue and visible blood. The mid-part of each muscle was mounted in embedding tissue (Tissue-Tek ${ }^{\circledR}$, Miles Laboratories), frozen in 2-methylbutane (Sigma-Aldrich, St. Louis, MO, USA), cooled in liquid $\mathrm{N}_{2}$, and stored at $-80{ }^{\circ} \mathrm{C}$ until further analysis were performed. Finally, animals were sacrificed by an intracardial injection of pentobarbital sodium.

Experiment 2: After termination of treadmill exercise, all rats were allowed to remain sedentary (day 11-17), enduring hindquarter paralyses (EAE group, day 11-17) and (partial) recovery (day 17). At day 13 and 17 additional arterial blood samples were collected from the tail and serum samples were stored at $-80{ }^{\circ} \mathrm{C}$. Finally, isokinetic foot extensor performance and muscle sampling were performed, whereupon animals were sacrificed.

During the complete study animal well-being was monitored. If an animal developed hindquarter paralysis 
earlier than expected, restricting the animal to run, the exercise program was immediately terminated, in accordance with the designated endpoint of exercise, where after the animal was excluded and humanely euthanized.

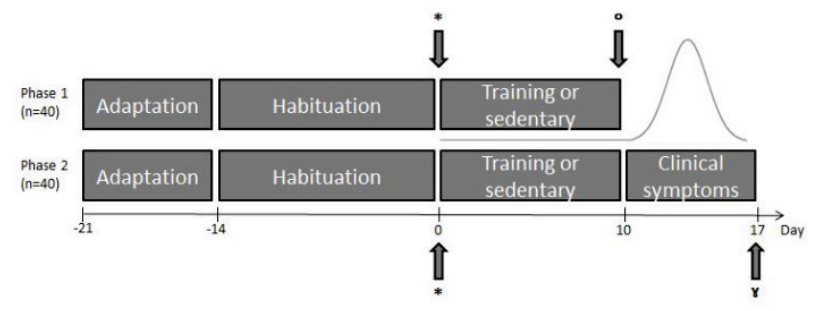

Fig. 1. Study design of the protocol. Following adaptation rats were familiarized to treadmill running during habituation. From the habituation period onwards, daily food intake and body weight were registered. After $\mathrm{EAE}$ induction $\left({ }^{*}\right), \mathrm{CON}^{\mathrm{EX}}$ and $\mathrm{EAE} \mathrm{E}^{\mathrm{EX}}$ rats were subjected to daily physical exercise, during 10 days. During experiment $1(n=40)$, isokinetic foot extensor performance was measured and muscle samples of EDL, TA and SOL were collected before onset of clinical symptoms $\left({ }^{\circ}\right)$. During experiment $2(n=40)$, isokinetic foot extensor performance and muscle sampling were performed after (partial) hindquarter paralysis recovery $(\curlyvee)$. Finally, all rats were sacrificed.

\section{EAE induction}

EAE was induced in EAE subgroups by a single percutaneous injection in both footpads (100 $\mu \mathrm{l} /$ foot) under isoflurane anesthesia (Polfliet et al. 2002) and consisted, per animal, of $24 \mu \mathrm{l}$ purified myelin basic protein (MBP, $25 \mathrm{mg} / \mathrm{ml}$ ) in combination with $25 \mu \mathrm{l}$ 7RA heat-killed mycobacterium tuberculosis $(20 \mathrm{mg} / \mathrm{ml}$, Difco), $120 \mu \mathrm{l}$ complete Freunds adjuvant (CFA, Difco) and $31 \mu 1$ phosphate-buffered saline (PBS).

\section{Primary outcome measures}

\section{Fiber CSA and proportion}

To quantify muscle fiber CSA and proportion of TA and EDL, serial transverse sections $(8 \mu \mathrm{m})$ from the obtained muscle samples were cut by an operator blinded to sample randomization at $-20{ }^{\circ} \mathrm{C}$ and stained by means of triple-staining. Briefly, sections were incubated during 45 min with a mix in PBS of 2 mouse monoclonal antibodies against myosin heavy chain I and IIa (1:25; A4.840 IgM supernatant and 1:25; N2.261 IgG $_{1}$ supernatant, respectively; Developmental Studies Hybridoma Bank, Iowa, USA) and 1 rabbit polyclonal laminin antibody (1:100; L-9393, Sigma, Zwijndrecht, The Netherlands). Next, the second incubation comprised a mixture of secondary antibodies (1:500, Goat antiMouse IgM AlexaFluor 555 [excitation/emission wavelength $\max 555 / 565 \mathrm{~nm}$ ]; 1:200 Goat anti-Mouse
$\mathrm{IgG}_{1}$ AlexaFluor 488 [excitation/emission wavelength $\max 495 / 519 \mathrm{~nm}]$ and 1:130 Goat anti-Rabbit IgG AlexaFluor 350 [excitation/emission wavelength $\max$ 346/442 nm]; Molecular probes, Invitrogen, Breda, The Netherlands), diluted in PBS. The fluorescence signals were recorded using a TRITC and FITC filter for type I and IIa muscle fibers, respectively, and a DAPI filter for cell membrane, using a Nikon E800 fluorescence microscope (Nikon, Badhoevedorp, The Netherlands). The regions that were not fluorescent, representing type IIx and IIb fibers, were grouped together and called type $\mathrm{IIx}+\mathrm{b}$ fibers. Digital images (x20 magnification, exposure time for TRITC and FITC $400 \mathrm{~ms}$, DAPI $800 \mathrm{~ms}$ ) were analyzed using NIS Elements software (Nikon, Badhoevedorp, The Netherlands).

Due to cross-reaction the above described protocol was not able to stain muscle fibers of SOL, therefore, a second technique was used to quantify fiber CSA and proportion of SOL. Serial transverse sections $(10 \mu \mathrm{m})$ from the obtained muscle samples were cut by an operator blinded to sample randomization at $-20^{\circ} \mathrm{C}$ and stained by means of ATPase histochemistry, after preincubation at $\mathrm{pH} 4.4,4.65$ and 10.3 , essentially following the procedure of Brooke and Kaiser (Brooke and Kaiser 1970). The serial sections were visualized and analyzed using a Leica DM2000 microscope (Leica, Stockholm, Sweden) and a Leica Hi-resolution Color DFC camera (Leica, Stockholm, Sweden) combined with image-analysis software (Leica Qwin ver. 3, Leica, Stockholm, Sweden). This software was able to automatically draw a fiber mask at the stained sections. Afterwards, this mask was fitted manually to the cell borders of selected fibers. Only fibers cut perpendicularly to their longitudinal axis were used for the determination of fiber size. In general, $5 \pm 2$ images were quantified per muscle section and $601 \pm 128$ fibers were included in CSA and fiber type analysis. Finally, muscle fiber characteristics are reported by means of CSA and fiber type proportion data.

\section{Hindquarter paralysis}

After EAE induction all EAE rats were examined daily at 8.30 a.m. for the development of hindquarter paralysis. Typically, hindquarter paralysis developed 12 to 14 days after induction, followed by partial recovery (day 17). Symptoms were blind-scored on a scale ranging from 0 to 5: 0 , no signs; 0.5 : partial loss of tail tonus (defined as the disease onset); 1.0: complete loss of tail tonus; 2.0 : hind limb paresis; 3.0: 
hind limb paralysis; 4.0: moribund; 5.0: death due to EAE (Polfliet et al. 2002). Disease peak was defined as the highest clinical score of each animal. The clinical endpoints were based on the clinical scores. If an animal exceeded a score of 4 , suffering complete paralysis of hind limbs and midriff the animal was excluded and humanely euthanized. If an animal was not able to eat or drink independently, the animal was excluded and euthanized as well. Overall hindquarter paralysis is expressed as the average of the daily scores per group.

\section{Secondary outcome measures}

\section{Isokinetic foot extensor performance}

After general anesthesia, left hind limb foot extensor muscle strength was assessed during fatiguing isokinetic muscle contractions, as described elsewhere (Hesselink et al. 1996, Komulainen et al. 2000). Briefly, percutaneous needle electrodes were placed on the common peroneal nerve fusing 130 consecutive concentric isokinetic foot extensions $(50 \%, \mathrm{~s}, 1 \mathrm{~mA}$, $250 \mathrm{~ms}, 3 \mathrm{~s}$ rest intervals) after standardized fixation of knee and ankle on a custom build Ashton-Miller like rat dynamometer (Ashton-Miller et al. 1992). Muscle performance of all animals is reported as muscle work $(\mathrm{mJ})$ over number of contractions.

\section{BDNF measurement}

Serum BDNF concentrations were blinded determined by electrochemoluminiscence ELISA using Meso Scale Discovery (MSD) plates spotted with BDNFspecific capture antibodies according to manufacturer's instructions (Meso Scale Discovery, USA). Briefly, after blocking of the plate, rats' sera and calibrators were dispensed into the MSD plate and incubated for two hours at room temperature. Calibrator was spiked in matrix like solution and used as positive control during the measurements. The plates were subsequently washed to remove unbound material and a BDNF-specific $1 \mathrm{X}$ MSD SULFOTAG-labelled detection antibody was dispensed into the MSD plate. Following a 2-h incubation step $2 \mathrm{X}$ read buffer $\mathrm{T}$ was added and plates were measured on the SECTOR $\AA$ Imager, expressing BDNF concentrations in $\mathrm{pg} / \mathrm{ml}$.

\section{Body weight and food intake}

Daily body weight and food intake was registered using a digital balance (Sartorius $\AA$, Germany) at 8 a.m. and expressed in $\mathrm{g}$.

\section{Statistical analyses}

All data were analyzed using either SAS software (SAS Institute Inc, Cary, USA) or R 2.15.2 software (Wood 2006). Additive and linear mixed models and unpaired student t-tests were used to analyze data. For the unpaired student t-tests, normality was checked using the Shapiro-Wilk test. For the generalized linear mixed model, diagnostics were based on the studentized residuals.

In particular, body weight and food intake were analyzed by a (Group [CON; EAE] x Activity [SED; EX] $x$ Time) mixed model ANOVA. To analyze the course of hindquarter paralysis, a (EAE Group [SED; EX] x Time [Days 0-17]) mixed model ANOVA was used. To analyze disease onset, which was defined as a hindquarter paralysis score equal to 0.5 , overall symptom intensity was compared between groups by an unpaired student's t-test. Muscle fiber type area and proportion were analyzed using a 2x2 (Group [CON; EAE] x Activity [SED; EX]) ANOVA. BDNF profiles were analyzed by a (Group [CON; EAE] x Activity [SED; EX] x Time) mixed model ANOVA. Muscle fatigue of isokinetic foot extensor data was analyzed using a (Group [CON; EAE] $x$ Activity [SED; EX] x Contraction [number of dynamic muscle contractions]) mixed model ANOVA. Furthermore, (generalized) additive mixed models were fitted using thin-plate regression splines to model the contraction effect and interactions with both the disease status and whether or not an intervention took place. Testing effects, starting with the interaction effect, was performed using approximate F-tests. Correlations were analyzed by means of Pearson's correlation analysis. All data are presented as mean \pm SEM and the threshold for statistical significance was set at $\mathrm{p}<0.05$.

\section{Results}

\section{Primary outcome measures}

Muscle fiber cross sectional area and proportion

Representative images from the muscle fiber analysis are shown in Figure 2. Muscle fiber CSA of the different muscles is represented in Figures 3 and 4. Compared to $\mathrm{CON}^{\mathrm{SED}}$, EAE reduced CSA of type I, IIa and IIx+b fibers of all muscles in both experiments. In experiment 1 , exercise did not affect CSA of the different fiber types of $\mathrm{CON}^{\mathrm{EX}}$, compared to $\mathrm{CON}^{\mathrm{SED}}$, except for type IIa muscle fiber CSA of SOL. Furthermore, in $\mathrm{EAE}^{\mathrm{EX}}$, compared to $\mathrm{EAE}^{\mathrm{SED}}$, exercise was able to increase fiber type IIa and IIx+b CSA in SOL and tended 
to increase mean CSA and muscle fiber type IIx $+\mathrm{b}$ CSA of TA $(p<0.1)$. In experiment 2 , exercise did not affect $\mathrm{CSA}$ of the different fiber types of $\mathrm{CON}^{\mathrm{EX}}$, compared to $\mathrm{CON}^{\mathrm{SED}}$, except for type IIa and IIx+b muscle fiber CSA of SOL. Furthermore, in $\mathrm{EAE}^{\mathrm{EX}}$, compared to $\mathrm{EAE}^{\mathrm{SED}}$, exercise increased mean CSA and muscle fiber type II $x+b$ CSA of TA, whereas other muscle fiber types remained unaffected by exercise.
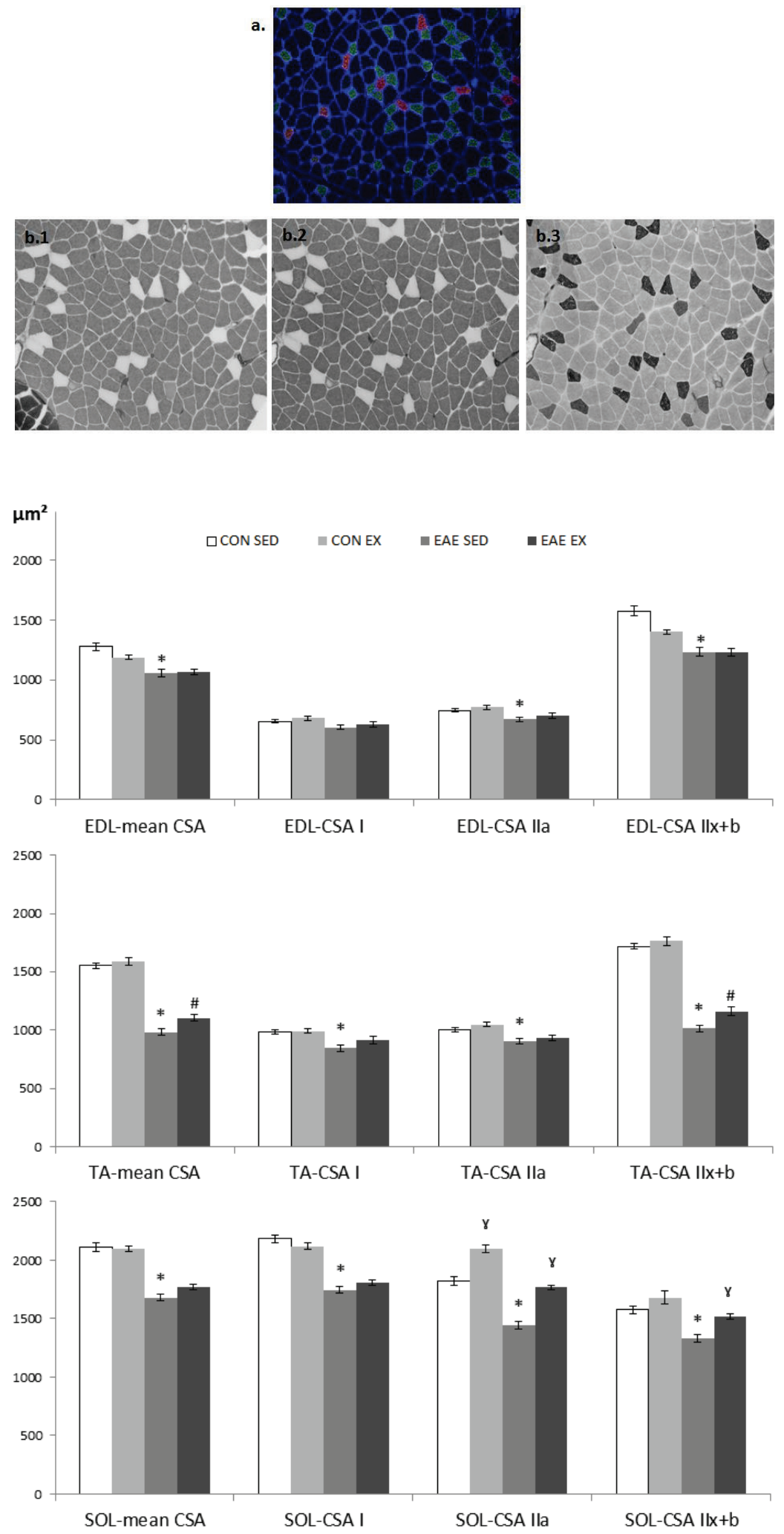

Fig. 2. Representative image of immunochemical staining (a) and ATPase histochemistry (b). a. Immunochemical staining to identify different muscle fiber types, in particular type I (red), type IIa (green) and type IIx $+b$ (not stained) muscle fibers of TA and EDL. b. ATPase histochemistry after preincubation at pH 4.4 (b.1), 4.65 (b.2) and 10.3 (b.3) to identify different muscle fibers of SOL. Muscle tissue samples of the right hind limb were collected, under anesthesia, before onset of clinical symptoms (experiment $1, n=40$ ) or after (partial) hindquarter paralysis recovery (experiment 2, $\mathrm{n}=40$ ). The mid-part of each muscle was mounted in cryoprotectant, frozen in 2-methylbutane, cooled in liquid $\mathrm{N}_{2}$, and stored at $-80^{\circ} \mathrm{C}$ until immunochemical staining and ATPase histochemistry were performed.

Fig. 3. Effect of $E A E$ and treadmill exercises on cross sectional area (CSA) of extensor digitorum longus (EDL), tibialis anterior (TA) and soleus (SOL), during experiment 1 , comprising 10 days of exercise. After immunochemical staining and ATPase histochemistry (Fig. 2), CSA of muscle fibers of EDL, TA and SOL were determined. Mean muscle fiber CSA, as well as type I, IIa and IIx+b CSA are represented separately for each muscle (EDL, TA and SOL) for all groups ( $n=10 /$ group). Data are presented as mean \pm SEM. * disease effect, comparison between CON $^{S E D}$ and EAE $^{\mathrm{SED}}$. $\gamma$ intervention effect, compared to corresponding SED group. \# $\mathrm{p} \geq 0.05$, but $<0.1$, intervention effect, compared to corresponding SED group. 

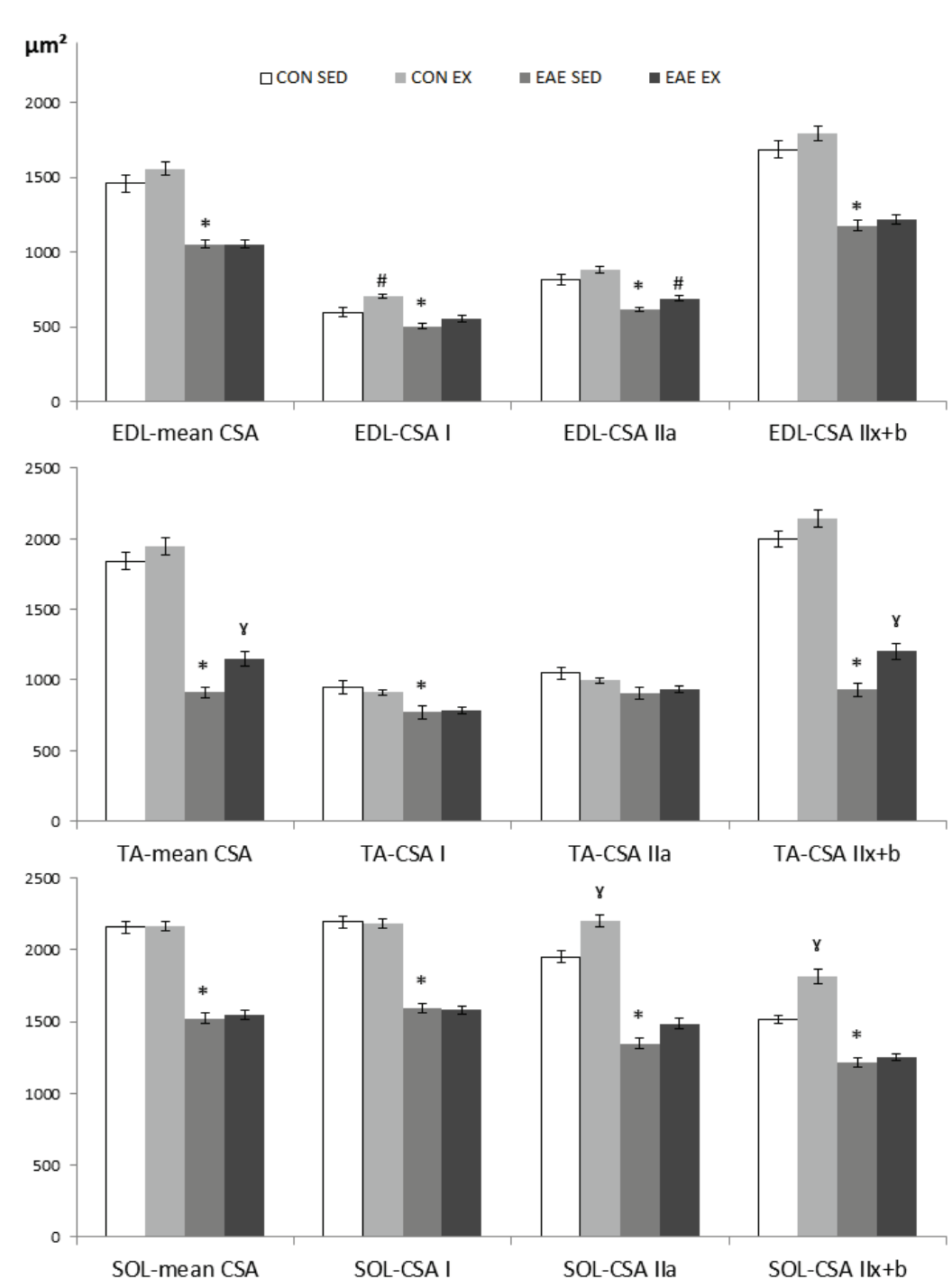

Fig. 4. Effect of $E A E$ and treadmill exercises on cross sectional area (CSA) of extensor digitorum longus (EDL), tibialis anterior (TA) and soleus (SOL), during experiment 2 , comprising 10 days of exercise and 7 sedentary days. After immunochemical staining and ATPase histochemistry (Fig. 2), CSA of muscle fibers of $E D L, T A$ and SOL were determined. Mean muscle fiber CSA, as well as type I, IIa and IIx+b CSA are represented separately for each muscle (EDL, TA and SOL) for all groups ( $n=10 /$ group). Data are presented as mean \pm SEM. * disease effect, comparison between $C N^{S E D}$ and $E A E^{S E D}$. $\gamma$ intervention effect, compared to corresponding SED group. \# $p \geq 0.05$, but $<0.1$, intervention effect, compared to corresponding SED group.
Muscle fiber proportions of the different muscles are represented in Figure 5. Compared to $\mathrm{CON}^{\mathrm{SED}}$, EAE decreased the proportion of EDL, TA and SOL type I fibers and increased the proportion of type IIx $+b$ of SOL in experiment 2, whereas during experiment 1 muscle fiber distribution remained unaffected by EAE. Furthermore, exercise did not affect the proportion of the different fiber types in experiment 1, except for type I distribution of SOL in $\mathrm{CON}^{\mathrm{EX}}$, which increased, compared to $\mathrm{CON}^{\mathrm{SED}}$. In experiment 2 exercise did not affect the proportion of the different fiber types in $\mathrm{CON}^{\mathrm{EX}}$. In $\mathrm{EAE}^{\mathrm{EX}}$ on the other hand, compared to $\mathrm{EAE}^{\mathrm{SED}}$, exercise increased the proportion of EDL and TA type I and IIa fibers, whereas the proportion of type $\mathrm{IIx}+\mathrm{b}$ fibers decreased after treadmill running in EDL, TA and SOL.

\section{Hindquarter paralysis (experiment 2)}

Overall, the hindquarter paralysis curve of $\mathrm{EAE}^{\mathrm{SED}}$ and $\mathrm{EAE}^{\mathrm{EX}}$ tended to differ over time (intervention $\mathrm{x}$ time, $\mathrm{p}=0.07)$. Moreover, exercise delayed the occurrence of the disease peak of $\mathrm{EAE}^{\mathrm{EX}}$, compared to $\mathrm{EAE}^{\mathrm{SED}}$, by $1.0 \pm 0.3$ days $(\mathrm{p}<0.05)$, whereas the severity of the disease peak $(2.4 \pm 0.3)$ was comparable between both groups. Compared to $\mathrm{EAE}^{\mathrm{SED}}$, hindquarter paralysis onset tended to be delayed by $0.7 \pm 0.4$ day in $\operatorname{EAE}^{\mathrm{EX}}$ ( $p=0.1$, Table 1). Furthermore, the degree of recovery at day 17 was comparable between both groups $(0.5 \pm 0.2)$. Finally, no animals reached the human endpoint (score $>4$ or inability to eat or drink independently).

\section{Secondary outcome measures \\ Isokinetic muscle strength}

In both experiments muscle work of $\mathrm{CON}$ peaked during the first 30 contractions, then declined and remained stable throughout the remaining contractions. In EAE, muscle work did not peak but was stable from onset (disease $x$ contraction, $p<0.05$, Fig. 6). 
Table 1. Disease onset and peak of $E A E^{\mathrm{SED}}$ and $E A E^{\mathrm{EX}}$ during experiment 2.

\begin{tabular}{|c|c|c|c|c|}
\hline & $\mathbf{E A E} \mathbf{E}^{\text {SED }}$ & $\mathbf{E A} \mathbf{E}^{\mathbf{E X}}$ & Difference & p-value \\
\hline \multicolumn{5}{|c|}{ Disease onset } \\
\hline Day & $11.2 \pm 0.3$ & $11.9 \pm 0.4$ & $0.7 \pm 0.4$ & 0.1 \\
\hline Score & 0.5 & 0.5 & / & NS \\
\hline \multicolumn{5}{|c|}{ Disease peak } \\
\hline Day & $13 \pm 0.3$ & $14 \pm 0.3$ & $1.0 \pm 0.3$ & 0.01 \\
\hline Score & $2.38 \pm 0.2$ & $2.38 \pm 0.3$ & / & NS \\
\hline
\end{tabular}

Data are presented as mean \pm SEM.
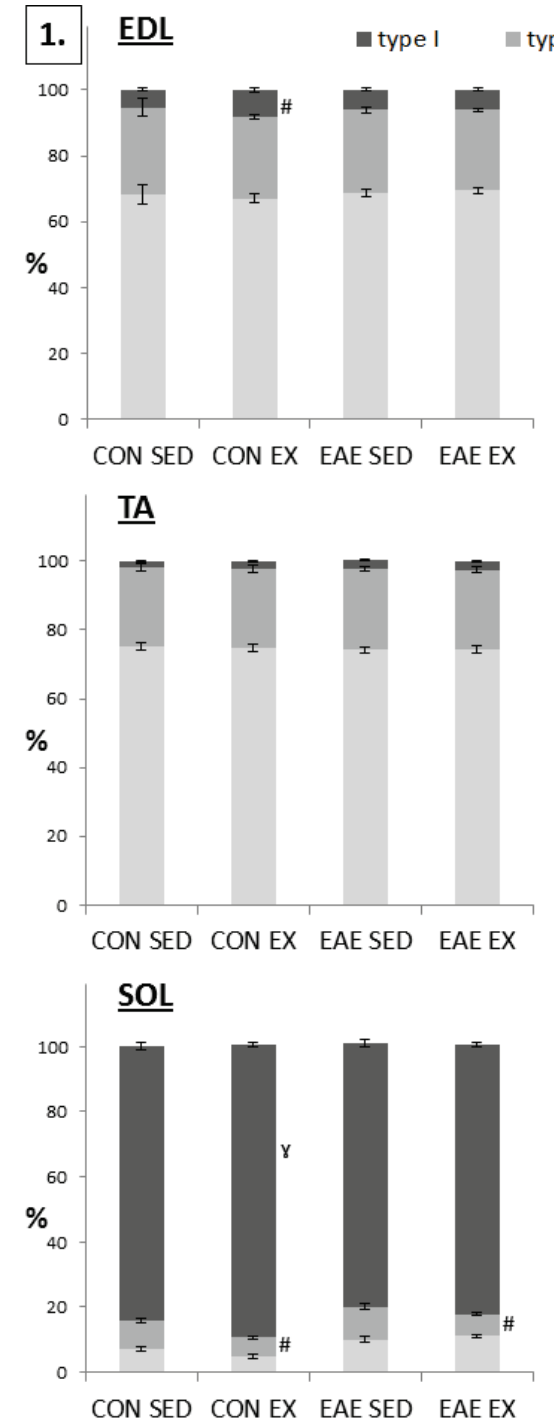
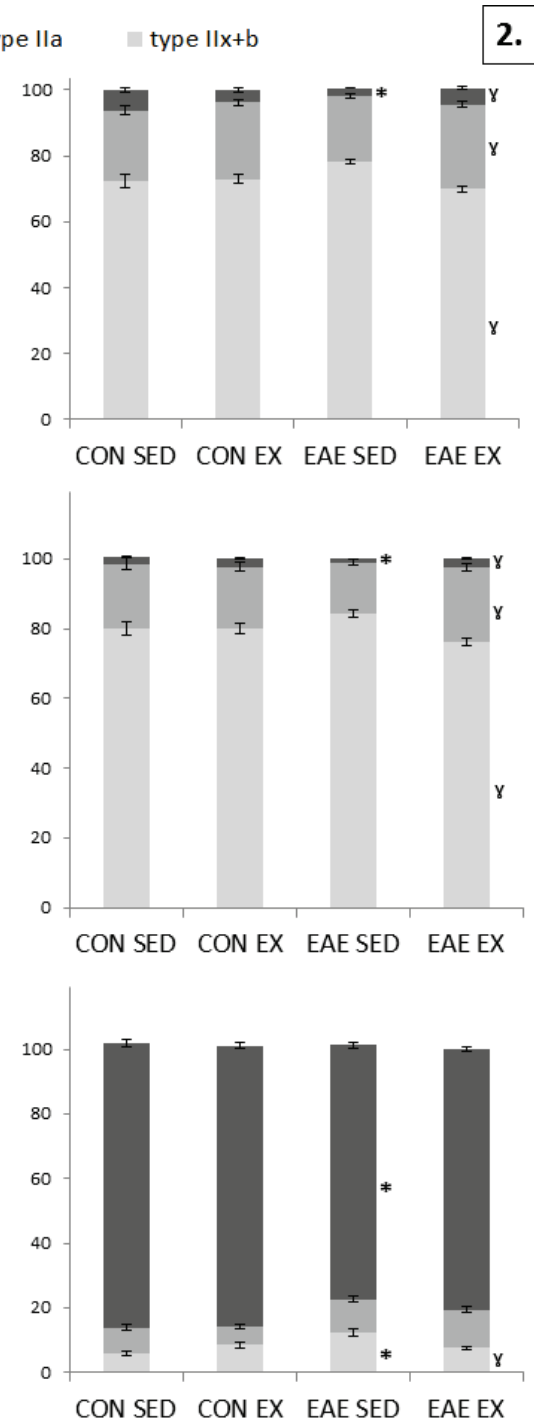

2. Fig. 5. Effect of EAE and treadmill exercises on muscle fiber proportion of extensor digitorum longus (EDL), tibialis anterior (TA) and soleus (SOL), during experiment 1 and 2 . After immunochemical staining and ATPase histochemistry (Fig. 2), muscle fiber proportion of EDL, TA and SOL were determined. Different proportions of muscle fiber types I, IIa and IIx+b are shown as percentages. Data are presented as mean \pm SEM. * disease effect, comparison between $\mathrm{CON}^{\mathrm{SED}}$ and $\mathrm{EAE}^{\mathrm{SED}}$. $\gamma$ intervention effect, compared to corresponding SED group. $\# p \geq 0.05$, but $<0.1$, intervention effect, compared to corresponding SED group.

\section{compared to corresponding SED group.}

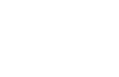

\section{$B D N F$ response}

During both experiments, BDNF concentrations of all groups increased over time $(\mathrm{p}<0.05)$. In particular, BDNF concentrations of all animals remained stable during the first 9 days, where after it increased during both experiments (Table 2 ).

Body weight and food intake

Body weight and food intake (Table 3) decreased immediately after EAE induction in both experiments, on average $\sim 10 \%$ and $\sim 65 \%$, respectively, $(\mathrm{p}<0.05)$. Hereafter, body weight and food intake recovered gradually until the onset of hindquarter 
paralysis on day 11. During hindquarter paralysis (experiment 2), body weight and food intake of EAE groups decreased, on average, $\sim 15 \%$ and $\sim 60 \%$, respectively $(p<0.05)$. Here, exercise was able to temper reduced food intake $(\mathrm{p}<0.05)$.

Table 2. BNDF concentrations (pg/ml), in CON and EAE animals, during experiment 1 and 2.

\begin{tabular}{|c|c|c|c|c|}
\hline BDNF conc $(\mathrm{pg} / \mathrm{ml})$ & $\mathrm{CON}^{\mathrm{SED}}$ & $\mathrm{CON}^{\mathrm{EX}}$ & EAE $E^{\text {SED }}$ & $\mathbf{E A E} \mathbf{E}^{\mathrm{EX}}$ \\
\hline \multicolumn{5}{|l|}{ Experiment 1} \\
\hline Day 0 & $1906 \pm 88$ & $1740 \pm 66$ & $1918 \pm 86$ & $1753 \pm 45$ \\
\hline Day 9 & $1843 \pm 78$ & $1683 \pm 89$ & $1593 \pm 67$ & $1619 \pm 86$ \\
\hline Day 11 & $2573 \pm 111 *$ & $2213 \pm 76^{*}$ & $2219 \pm 89^{*}$ & $2031 \pm 79^{*}$ \\
\hline \multicolumn{5}{|l|}{ Experiment 2} \\
\hline Day 0 & $1468 \pm 220$ & $1717 \pm 202$ & $1785 \pm 227$ & $1597 \pm 227$ \\
\hline Day 9 & $1880 \pm 126$ & $1775 \pm 112$ & $1790 \pm 70$ & $1748 \pm 104$ \\
\hline Day 13 & $2468 \pm 265$ & $2379 \pm 194$ & $2428 \pm 165$ & $2394 \pm 239$ \\
\hline Day 17 & $2508 \pm 235^{*}$ & $2696 \pm 277 *$ & $2484 \pm 147 *$ & $2603 \pm 225^{*}$ \\
\hline
\end{tabular}

Data are presented as mean \pm SEM. * within group effect.
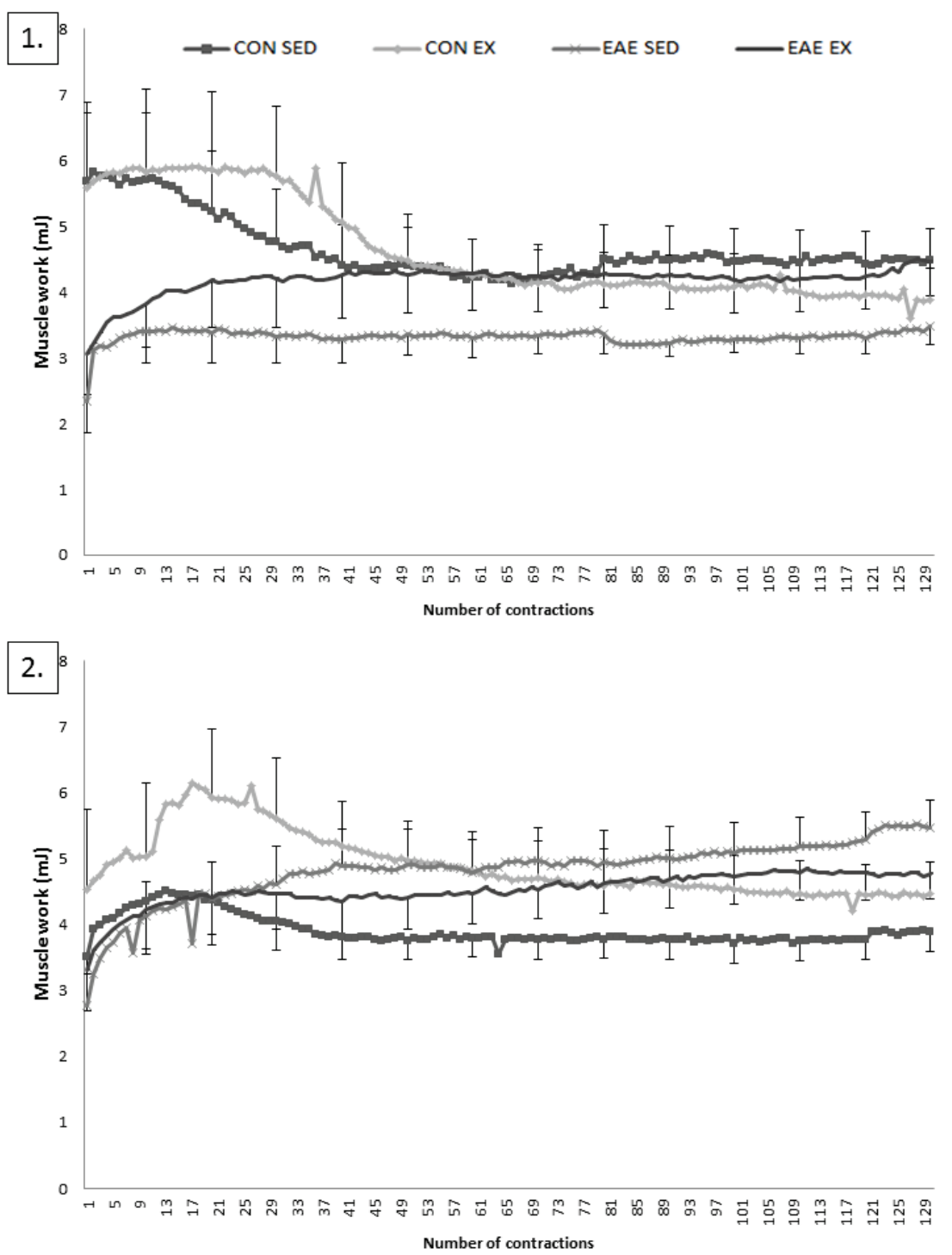

Fig. 6. Isokinetic muscle work in healthy and EAE rats, during experiment 1 and 2. Before onset of clinical symptoms (experiment 1 , $\mathrm{n}=40$ ) or after (partial) hindquarter paralysis recovery (experiment $2, \mathrm{n}=40$ ) repetitive isokinetic foot extensor performance of the left hind limb was measured under anesthesia. Values are mean \pm SEM and express muscle work $(\mathrm{Nm})$ during 130 consecutive maximal muscle contractions (1 $\mathrm{mA}, 150 \mathrm{~Hz}, 250 \mathrm{~ms}$ ) in CON and EAE rats. 


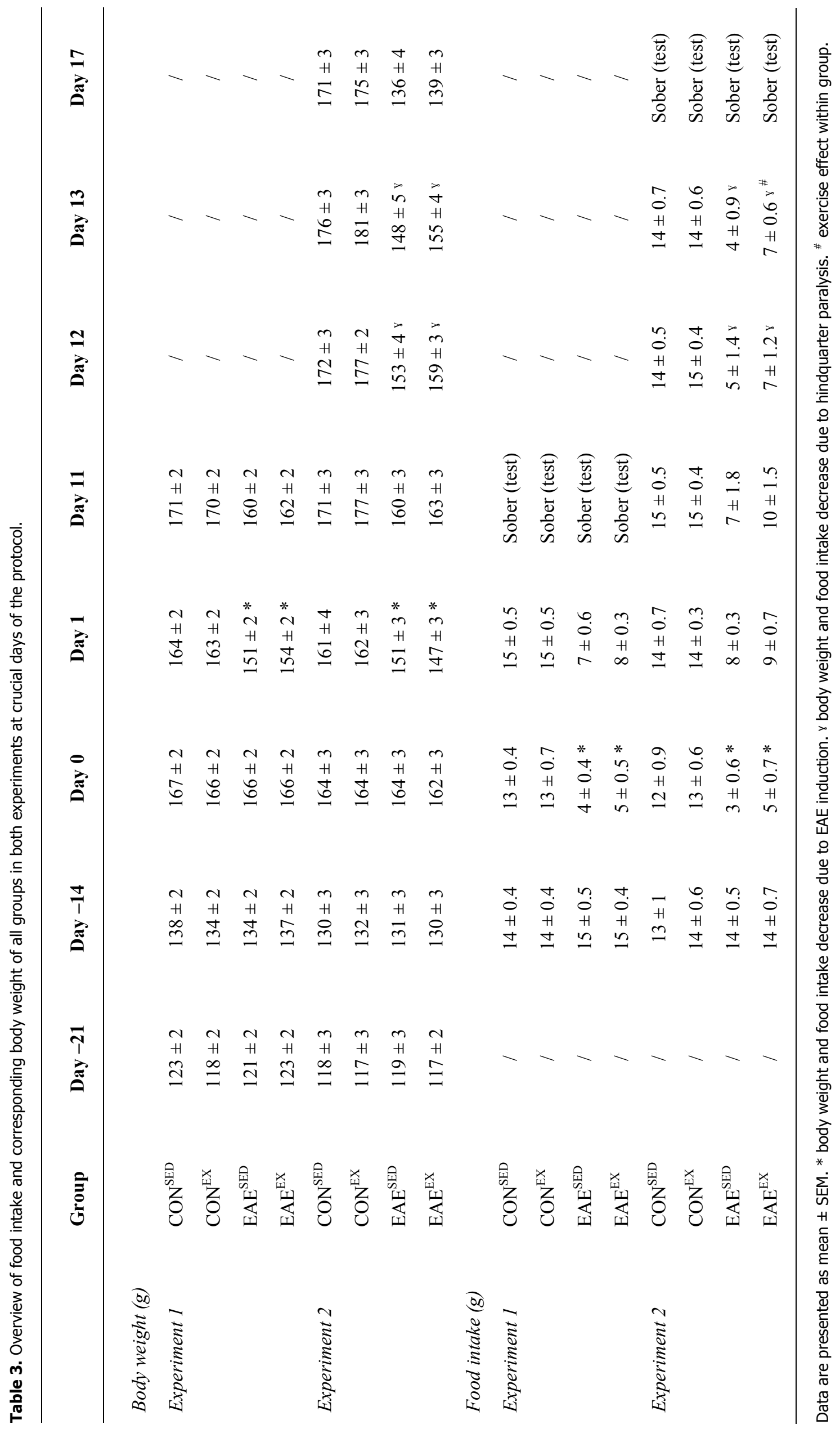




\section{Correlations}

In EAE groups, mean CSA of TA and SOL were negatively correlated with the disease peak (TA: $\mathrm{r}=-0.60$ and SOL: $\mathrm{r}=-0.63, \mathrm{p}<0.05)$, as well as TA fiber IIx $+\mathrm{b}$ CSA, SOL fiber I and IIa CSA $(r=-0.58$, $-0.64,-0.51$, respectively, $\mathrm{p}<0.05)$. Furthermore, TA mean CSA and TA fiber type IIx $+\mathrm{b}$ CSA were positively correlated with the day of the disease onset $(r=0.52$ and 0.49 , respectively, $\mathrm{p}<0.05$ ). CSA of EDL and BDNF profiles did not correlate with disease onset or disease peak in EAE.

\section{Drop out}

In total, one EAE animal died, unrelated to EAE, during experiment 1 of the study. Overall, there were no significant differences in survival rates between groups.

\section{Discussion}

This study investigated the impact of high intensity treadmill exercise on muscle morphology, disease course and BDNF release in EAE animals, immediately before the onset (experiment 1) and after (experiment 2) hindquarter paralysis. During both experiments, EAE reduced mean fiber CSA, as well as type I, IIa and IIx+b fiber CSA in TA, EDL and SOL. Under the conditions of the present study exercise was, in general, not able to prevent muscle fiber atrophy in both experiments, with the exception of some minor changes in $\mathrm{CON}^{\mathrm{EX}}$ and $\mathrm{EAE}^{\mathrm{EX}}$. Muscle work of $\mathrm{CON}$ peaked during the first 30 contractions and then progressively declined during the remaining contractions, while muscle work of EAE remained stable. High intensity exercise delayed hindquarter paralysis peak. Finally, the present study indicated that the BDNF response was not affected by EAE or exercise.

Investigations of high-intensity exercise to ameliorate the progressive muscle wasting, associated with a high proportion of MS patients, are highly relevant to evaluate the potential of rehabilitation. In laboratory settings these investigations are commonly based on the EAE model, however, the emerging data are difficult to interpret, due in part to the different modalities used, such as aerobic or swimming exercise. Additionally the use of different EAE variants and protocols, such as initiation of the exercise regimen prior or subsequent to EAE induction, confound the comparison of data. The selection of treadmill running exercise for the present studies was based on previous research from our laboratory demonstrating that whilst low $(5 \mathrm{~m} / \mathrm{min}$, $0^{\circ}$ inclination) and moderate $\left(11 \mathrm{~m} / \mathrm{min}, 15^{\circ}\right.$ inclination) intensity treadmill/running exercise were not able to delay EAE associated hindquarter paralysis, high intensity (18 $\mathrm{m} / \mathrm{min}, 25^{\circ}$ inclination) exercise was (Wens et al. 2015). A further consideration was that swimming exercise may cause excessive stress to the animals for the induction of favorable adaptations in muscle fiber characteristics to exercise. Furthermore, research investigating the influence of exercise on muscle morphology or muscle fiber characteristics frequently applies treadmill or voluntary running at mild-tomoderate intensity, which also justifies our choice of exercise modality.

Results obtained in this study should be translated with caution to MS patients. Nevertheless, the results of this study may provide important information, which would be expected to be most reflective on mildto-moderate and relapsing remitting MS, regarding the use of regular exercise prior to the start and at the very early phase of the disease, where medical treatment in MS patients have been proven crucial (Comi et al. 2013). Also, the present study provides important indications and may be useful to investigate motor function/muscle morphology, MS progression and management of the disease during and after high intensity aerobic exercise. In doing so, we anticipate to further reveal the full potential of exercise therapy in the treatment of MS in general and motor function in MS in particular.

\section{EAE and hindquarter paralysis}

The applied high intensity aerobic exercise was able to delay hindquarter paralysis peak and tended to delay hindquarter paralysis onset. This was mirrored by a reduced food intake drop during paralysis. These findings confirm our previously reported results in EAE animals (Broekmans et al. 2009). Also, other authors reported delayed onset of clinical EAE symptoms (Le Page et al. 1994, 1996, Rossi et al. 2009). However, it remains difficult to compare studies due to the application of different exercise protocols in terms of exercise intensity, volume and type (voluntary exercise versus quantified training load and intensity). Based on other literature, it is fair to assume that exercise intensity was near the anaerobic threshold (Cunha et al. 2009, Voltarelli et al. 2002), which is probably higher, compared to the work of Le Page et al. (1994, 1996) and Rossi et al. (2009). However, lactate concentrations and $\mathrm{VO}_{2}$ kinetics were not measured. As such, our present 
and former findings and the above-described work of others suggest that exercise intensity is the key determinant of the impact of exercise training on the course of EAE. Moreover, it would be interesting to investigate this hypothesis in MS patients.

\section{Muscle morphology and exercise in EAE}

Muscle morphology was also investigated in this rat strain, since Lewis rats appear to be the most specialized laboratory rodents in respect to skeletal muscle composition, as their SOL is the slowest and their EDL is the fastest among other rat strains. SOL is an antigravity muscle, designed to sustain prolonged activity, whereas EDL (and TA) is a fast muscle involved in short intermittent bursts (Novak et al. 2010). Comparable to results reported by others (Novak et al. 2010, Soukup et al. 2002, 2009), this study showed that SOL is composed of a great majority of slow type I fibers and a variable, but low, number of fast type IIa fibers, whereas EDL (and TA) is composed of a low number of type I fibers and of a variable proportion of (a medium number of) IIa and (a majority of) II $x+b$ fibers.

The present study also showed that CSA of all muscle fiber types in EDL, TA and SOL decreased due to EAE. Our group (Broekmans et al. 2009, Eijnde et al. 2008, Wens et al. 2015) and De Haan et al. (2004), who investigated the impact of $\mathrm{EAE}$ on the medial gastrocnemius, previously reported similar results.

It is clear that treadmill running is able to reverse muscle fiber atrophy in other rat populations (Itai et al. 2004, Tanaka et al. 2004, Thompson 2002). In the present study, however, exercise was, in general, not able to affect either CSA or fiber proportion significantly, with some minor exceptions in, mainly, SOL. Interestingly, it was expected that an exercise effect in experiment 1 would be seen by excluding the tempering effect of the sedentary week of experiment 2 . However, the expected exercise effect was absent in all CON and EAE groups, suggesting that the applied exercise duration (10 days) was too short to induce the hypothesized muscle fiber alterations. Furthermore, this may imply that aerobic exercise is not optimal to induce adaptations in muscle fiber CSA and proportion in EAE rats. Therefore it is suggested to investigate the influence of resistance training on muscle fiber characteristics, since EAE is able to reduce type $\mathrm{IIx}+\mathrm{b}$ fiber CSA, which (in humans) are more susceptible to resistance training (Hornberger Jr. and Farrar 2004, Tamaki et al. 1997, Yarasheski et al. 1990).

\section{Muscle work and exercise in EAE}

Muscle work of CON animals peaked, during the first 30 contractions and then progressively declined, whereas muscle work of EAE remained stable. These findings confirm our previously reported results in EAE and CON animals (Eijnde et al. 2008, Wens et al. 2015). However, these results contradicts data reported by De Haan and co-workers (2004), who reported that muscle work of both CON and EAE peaked and then declined during a series of repeated isometric maximal contractions of $m$. gastrocnemius. The difference can, possibly, be explained by the fact that different muscle groups were analyzed. Moreover, TA and EDL having predominately glycolytic muscle fibers (Minnaard et al. 2005) in the present study versus medial gastrocnemius having a proximal region, containing all muscle fibers, and a distal region, containing only type IIx and IIb fibers (De Haan et al. 2004). Because fast glycolytic type IIb muscle fibers largely contribute to peak force production (Burke et al. 1973, Cotter et al. 1989) decreased CSA of type IIx $+b$ fibers probably explains the absence of peak muscle work in EAE during the first 30 repetitive isokinetic contractions. As mentioned earlier, it was expected that an exercise-induced effect in experiment 1 would be present by excluding the tempering effect of the sedentary week. Moreover, in experiment 1, muscle work results suggested an exercise-induced effect in both CON and EAE groups. In experiment 2, on the other hand, muscle work curves only suggested an exercise effect in CON. However, statistical significance was not reached in both experiments due to large variations between muscle work of different animals. Therefore, future research should include larger sample sizes to increase power.

\section{$B D N F$ and exercise in EAE}

Since BDNF is a neuroprotective mediator during remyelination after a relapse (Frota et al. 2009, Sarchielli et al. 2002) and because it is suggested that BDNF could play an important role in the therapeutic effect of exercise in MS (Ferris et al. 2007, Gold et al. 2003, Lewin and Barde 1996, Rojas et al. 2006), an elevation of the BDNF levels in the exercising rats was expected, as reported by others (Bernardes et al. 2013). However, BDNF profiles did not differ between groups, suggesting that the improved clinical parameters (delay of EAE onset and peak) are ameliorated by exercise per se and not by elevated BDNF-induced neuroplasticity. These findings are in accordance with recent research, 
investigating the effect of a 10-day forced treadmill exercise on neurotrophic factors in EAE and healthy animals (Patel and White 2013). The lack of difference between sedentary and exercised animals can possibly be explained by the short duration of the exercise program in our study and in the study of Patel and White (2013). In MS it is already proven that 8 weeks of exercise is not able to change BDNF levels (Castellano and White 2008, Schulz et al. 2004). These findings make it interesting to investigate the effect of a long term exercise program in EAE and MS. Furthermore, contradictory to our hypothesis, BDNF profiles of all groups increased. This could be possibly explained by the exposure of stress and elevated levels of stress hormones, since all animals experienced the same level of stress during the study. Research already demonstrated that short periods (15-60 min) of stress could induce an increased BDNF mRNA expression, leading to elevated BDNF protein concentrations and suggesting some degree of neuronal plasticity to deal with new stimuli (Marmigere et al. 2003, Rage et al. 2002). However, stress hormone concentrations were not measured during the present study. Therefore, it is recommended to further investigate the influence of stress hormones in the future. Finally, since there are no differences between CON and EAE, nor between SED and EX BDNF profiles, our results suggest that high intensity aerobic exercise does not worsen the disease, indicating that high intensity aerobic exercise is tolerable in EAE and possibly also tolerable in MS.

\section{Limitations and future directions}

The present study had some limitations, resulting in a few recommendations for future research. The lack of an explicit exercise effect, could possibly be explained by the short duration of the applied exercise program. Furthermore, aerobic exercise was perhaps not the optimal exercise modality to induce improvements of the investigated parameters in EAE rats. Therefore, it is suggested to investigate the long term effect of physical exercise in chronic EAE and the influence of resistance training on muscle contractile properties and BDNF release in EAE rats.

Furthermore, since the optimal exercise intensity is not known yet, it is recommended to quantify exercise intensity in future studies, measuring lactate concentrations and/or $\mathrm{VO}_{2}$ kinetics. Next, since forced treadmill running can induce stress, which could influence the EAE symptoms, it is recommended to measure stress hormone levels in future research.

\section{Conclusion}

In conclusion, the present study demonstrates that EAE reduces muscle fiber CSA of TA, EDL and SOL, which could in part explain the absence of peak muscle work during the first 30 contractions of isokinetic muscle performance in EAE animals. Furthermore, exercise increases muscle fiber type IIa and IIx $+\mathrm{b}$ CSA in SOL and is able to delay the onset and peak of EAE induced hindquarter paralysis. Finally, there was no difference between BDNF profiles of CON and EAE, suggesting that high intensity exercise does not worsen the disease.

\section{Conflict of Interest}

Ulrik Dalgas has received research support, travel grants and/or teaching honorary from Biogen Idec, Merck Serono and Sanofi Aventis and further serves as PI for the ongoing Biogen sponsored ACTIMS study.

\section{Acknowledgement}

The authors would like to thank Nathalie Cools and Nathalie Deckx of Antwerp University for providing skilled assistance during the BDNF measurements, Gitte Hartvigsen and Janni Mosgaard Jensen of Aarhus University for providing qualified assistance during the ATPase staining of soleus biopsies and Devid Muys, without whose help and support this study would not been possible.

This research received no grant from any funding agency in the public, commercial, or not-for-profit sectors.

\section{References}

ASHTON-MILLER JA, HE Y, KADHIRESAN VA, MCCUBBREY DA, FAULKNER JA: An apparatus to measure in vivo biomechanical behavior of dorsi- and plantarflexors of mouse ankle. J Appl Physiol 72: 1205-1211, 1992.

BEN-NUN A, WEKERLE H, COHEN IR: The rapid isolation of clonable antigen-specific T lymphocyte lines capable of mediating autoimmune encephalomyelitis. Eur J Immunol 11: 195-199, 1981. 
BEN-NUN A, COHEN IR: Experimental autoimmune encephalomyelitis (EAE) mediated by T cell lines: process of selection of lines and characterization of the cells. J Immunol 129: 303-308, 1982.

BERNARDES D, OLIVEIRA-LIMA OC, SILVA TV, FARACO CC, LEITE HR, JULIANO MA, SANTOS DM, BETHEA JR, BRAMBILLA R, ORIAN JM, ARANTES RM, CARVALHO-TAVARES J: Differential brain and spinal cord cytokine and BDNF levels in experimental autoimmune encephalomyelitis are modulated by prior and regular exercise. J Neuroimmunol 264: 24-34, 2013.

BROEKMANS T, HENDRIKS J, STINISSEN P, EIJNDE B: Physical exercise during acute EAE may affect clinical symptom progression. Mult Scler 15: S261-S262, 2009.

BROEKMANS T, ROELANTS M, ALDERS G, FEYS P, THIJS H, EIJNDE BO: Exploring the effects of a 20-week whole-body vibration training programme on leg muscle performance and function in persons with multiple sclerosis. J Rehabil Med 42: 866-872, 2010.

BROEKMANS T, ROELANTS M, FEYS P, ALDERS G, GIJBELS D, HANSSEN I, STINISSEN P, EIJNDE BO: Effects of long-term resistance training and simultaneous electro-stimulation on muscle strength and functional mobility in multiple sclerosis. Mult Scler 17: 468-477, 2011.

BROOKE MH, KAISER KK: Muscle fiber types: how many and what kind? Arch Neurol 23: 369-379, 1970.

BURKE RE, LEVINE DN, TSAIRIS P, ZAJAC FE: III Physiological types and histochemical profiles in motor units of the cat gastrocnemius. $J$ Physiol 234: 723-748, 1973.

CARROLL CC, GALLAGHER PM, SEIDLE ME, TRAPPE SW: Skeletal muscle characteristics of people with multiple sclerosis. Arch Phys Med Rehabil 86: 224-229, 2005.

CASTELLANO V, WHITE LJ: Serum brain-derived neurotrophic factor response to aerobic exercise in multiple sclerosis. J Neurol Sci 269: 85-91, 2008.

COLLETT J, DAWES H, MEANEY A, SACKLEY C, BARKER K, WADE D, IZARDI H, BATEMAN J, DUDA J, BUCKINGHAM E: Exercise for multiple sclerosis: a single-blind randomized trial comparing three exercise intensities. Mult Scler 17: 594-603, 2011.

COMI G, MARTINELLI V, RODEGHER M, MOIOLA L, LEOCANI L, BAJENARU O, CARRA A, ELOVAARA I, FAZEKAS F, HARTUNG HP, HILLERT J, KING J, KOMOLY S, LUBETZKI C, MONTALBAN X, MYHR KM, PREZIOSA P, RAVNBORG M, RIECKMANN P, ROCCA MA, WYNN D, YOUNG C, FILIPPI M: Effects of early treatment with glatiramer acetate in patients with clinically isolated syndrome. Mult Scler 19: 1074-1083, 2013.

COMPSTON A, COLES A: Multiple sclerosis. Lancet 359: 1221-1231, 2002.

CONSTANTINESCU CS, FAROOQI N, O'BRIEN K, GRAN B: Experimental autoimmune encephalomyelitis (EAE) as a model for multiple sclerosis (MS). Br J Pharmacol 164: 1079-1106, 2011.

COTMAN CW, BERCHTOLD NC: Exercise: a behavioral intervention to enhance brain health and plasticity. Trends Neurosci 25: 295-301, 2002.

COTTER M, CAMERON NE, LEAN DR, ROBERTSON S: Effects of long-term streptozotocin diabetes on the contractile and histochemical properties of rat muscles. J Exp Physiol 74: 65-74, 1989.

CUNHA RR, CUNHA VN, SEGUNDO PR, MOREIRA SR, KOKUBUN E, CAMPBELL CS, DE OLIVEIRA RJ, SIMOES HG: Determination of the lactate threshold and maximal blood lactate steady state intensity in aged rats. Cell Biochem Funct 27: 351-357, 2009.

DALGAS U, STENAGER E: Exercise and disease progression in multiple sclerosis: can exercise slow down the progression of multiple sclerosis? Ther Adv Neurol Disord 5: 81-95, 2012.

DALGAS U, STENAGER E, INGEMANN-HANSEN T: Multiple sclerosis and physical exercise: recommendations for the application of resistance-, endurance- and combined training. Mult Scler 14: 35-53, 2008.

DALGAS U, INGEMANN-HANSEN T, STENAGER E: Physical exercise and MS recommendations. Int MS $J$ 16: 5-11, 2009a.

DALGAS U, STENAGER E, JAKOBSEN J, PETERSEN T, HANSEN HJ, KNUDSEN C, OVERGAARD K, INGEMANN-HANSEN T: Resistance training improves muscle strength and functional capacity in multiple sclerosis. Neurology 73: 1478-1484, 2009b.

DALGAS U, STENAGER E, JAKOBSEN J, PETERSEN T, OVERGAARD K, INGEMANN-HANSEN T: Muscle fiber size increases following resistance training in multiple sclerosis. Mult Scler 16: 1367-1376, 2010. 
DE HAAN A, DE RUITER CJ, VAN DER WOUDE LH, JONGEN PJ: Contractile properties and fatigue of quadriceps muscles in multiple sclerosis. Muscle Nerve 23: 1534-1541, 2000.

DE HAAN A, VAN DER VLIET MR, HENDRIKS JJ, HEIJNEN DA, DIJKSTRA CD: Changes in characteristics of rat skeletal muscle after experimental allergic encephalomyelitis. Muscle Nerve 29: 369-375, 2004.

EIJNDE BO, BROCKMANS T, ALDERS G, HENDRIKS JJ, STINISSEN P: Muscle fatigue resistance during experimental autoimmune encephalomyelitis in rats. Mult Scler 14: S121, 2008.

FERRIS LT, WILLIAMS JS, SHEN CL: The effect of acute exercise on serum brain-derived neurotrophic factor levels and cognitive function. Med Sci Sports Exerc 39: 728-734, 2007.

FROTA ER, RODRIGUES DH, DONADI EA, BRUM DG, MACIEL DR, TEIXEIRA AL: Increased plasma levels of brain derived neurotrophic factor (BDNF) after multiple sclerosis relapse. Neurosci Lett 460: 130-132, 2009.

GARNER DJ, WIDRICK JJ: Cross-bridge mechanisms of muscle weakness in multiple sclerosis. Muscle Nerve 27: 456-464, 2003.

GENAIN CP, HAUSER SL: Allergic encephalomyelitis in common marmosets: pathogenesis of a multiple sclerosislike lesion. Methods 10: 420-434, 1996.

GOLD SM, SCHULZ KH, HARTMANN S, MLADEK M, LANG UE, HELLWEG R, REER R, BRAUMANN KM, HEESEN C: Basal serum levels and reactivity of nerve growth factor and brain-derived neurotrophic factor to standardized acute exercise in multiple sclerosis and controls. J Neuroimmunol 138: 99-105, 2003.

HESSELINK MK, KUIPERS H, GEURTEN P, VAN STRAATEN H: Structural muscle damage and muscle strength after incremental number of isometric and forced lengthening contractions. J Muscle Res Cell Motil 17: 335-341, 1996.

HORNBERGER TA JR, FARRAR RP: Physiological hypertrophy of the FHL muscle following 8 weeks of progressive resistance exercise in the rat. Can J Appl Physiol 29: 16-31, 2004.

HORTOBAGYI T, DEMPSEY L, FRASER D, ZHENG D, HAMILTON G, LAMBERT J, DOHM L: Changes in muscle strength, muscle fibre size and myofibrillar gene expression after immobilization and retraining in humans. J Physiol 524: 293-304, 2000.

INNES JR: Experimental "allergic" encephalitis: attempts to produce the disease in sheep and goats. J Comp Pathol 61: 241-250, 1951.

ITAI Y, KARIYA Y, HOSHINO Y: Morphological changes in rat hindlimb muscle fibres during recovery from disuse atrophy. Acta Physiol Scand 181: 217-224, 2004.

KENT-BRAUN JA, SHARMA KR, WEINER MW, MILLER RG: Effects of exercise on muscle activation and metabolism in multiple sclerosis. Muscle Nerve 17: 1162-1169, 1994.

KENT-BRAUN JA, NG AV, CASTRO M, WEINER MW, GELINAS D, DUDLEY GA, MILLER RG: Strength, skeletal muscle composition, and enzyme activity in multiple sclerosis. J Appl Physiol 83: 1998-2004, 1997.

KOMULAINEN J, KALLIOKOSKI R, KOSKINEN SO, DROST MR, KUIPERS H, HESSELINK MK: Controlled lengthening or shortening contraction-induced damage is followed by fiber hypertrophy in rat skeletal muscle. Int J Sports Med 21: 107-112, 2000.

LE PAGE C, FERRY A, RIEU M: Effect of muscular exercise on chronic relapsing experimental autoimmune encephalomyelitis. J Appl Physiol 77: 2341-2347, 1994.

LE PAGE C, BOURDOULOUS S, BERAUD E, COURAUD PO, RIEU M, FERRY A: Effect of physical exercise on adoptive experimental auto-immune encephalomyelitis in rats. Eur J Appl Physiol Occup Physiol 73: 130-135, 1996.

LEWIN GR, BARDE YA: Physiology of the neurotrophins. Annu Rev Neurosci 19: 289-317, 1996.

LIPTON MM, FREUND J: Allergic encephalomyelitis in the rat induced by the intracutaneous injection of central nervous system tissue and adjuvants. J Immunol 71: 98-109, 1953.

LU B: BDNF and activity-dependent synaptic modulation. Learn Mem 10: 86-98, 2003.

MARMIGERE F, GIVALOIS L, RAGE F, ARANCIBIA S, TAPIA-ARANCIBIA L: Rapid induction of BDNF expression in the hippocampus during immobilization stress challenge in adult rats. Hippocampus 13: 646-655, 2003.

MCALLISTER AK, KATZ LC, LO DC: Neurotrophins and synaptic plasticity. Annu Rev Neurosci 22: 295-318, 1999. 
MINNAARD R, DROST MR, WAGENMAKERS AJ, VAN KRANENBURG GP, KUIPERS H, HESSELINK MK: Skeletal muscle wasting and contractile performance in septic rats. Muscle Nerve 31: 339-348, 2005.

MORRISON LR: Disseminated encephalomyelitis experimentally produced by the use of homologous antigen. Arch Neurol Psychiatry 58: 391-416, 1947.

NG AV, MILLER RG, GELINAS D, KENT-BRAUN JA: Functional relationships of central and peripheral muscle alterations in multiple sclerosis. Muscle Nerve 29: 843-852, 2004.

NOSEWORTHY JH, LUCCHINETTI C, RODRIGUEZ M, WEINSHENKER BG: Multiple sclerosis. $N$ Engl J Med 343: 938-952, 2000.

NOVAK P, ZACHAROVA G, SOUKUP T: Individual, age and sex differences in fiber type composition of slow and fast muscles of adult Lewis rats: comparison with other rat strains. Physiol Res 59: 783-801, 2010.

OLITSKY PK, YAGER RH: Experimental disseminated encephalomyelitis in white mice. $J$ Exp Med 90: 213-224, 1949.

PATEL DI, WHITE LJ: Effect of 10-day forced treadmill training on neurotrophic factors in experimental autoimmune encephalomyelitis. Appl Physiol Nutr Metab 38: 194-199, 2013.

PETAJAN JH, WHITE AT: Recommendations for physical activity in patients with multiple sclerosis. Sports Med 27: 179-191, 1999.

POLFLIET MM, VAN DE VEERDONK, DOPP EA, VAN KESTEREN-HENDRIKX EM, VAN ROOIJEN N, DIJKSTRA CD, VAN DEN BERG TK: The role of perivascular and meningeal macrophages in experimental allergic encephalomyelitis. J Neuroimmunol 122: 1-8, 2002.

RAGE F, GIVALOIS L, MARMIGERE F, TAPIA-ARANCIBIA L, ARANCIBIA S: Immobilization stress rapidly modulates BDNF mRNA expression in the hypothalamus of adult male rats. Neuroscience 112: 309-318, 2002.

RAINE CS, TRAUGOTT U: Experimental autoimmune demyelination. Chronic relapsing models and their therapeutic implications for multiple sclerosis. Ann N Y Acad Sci 436: 33-51, 1984.

RIETBERG MB, BROOKS D, UITDEHAAG BM, KWAKKEL G: Exercise therapy for multiple sclerosis. Cochrane Database Syst Rev: CD003980, 2005.

ROJAS VS, STRUDER HK, VERA WB, SCHMIDT A, BLOCH W, HOLLMANN W: Acute BDNF and cortisol response to low intensity exercise and following ramp incremental exercise to exhaustion in humans. Brain Res 1121: 59-65, 2006.

ROSSI C, ANGELUCCI A, COSTANTIN L, BRASCHI C, MAZZANTINI M, BABBINI F, FABBRI ME, TESSAROLLO L, MAFFEI L, BERARDI N, CALEO M: Brain-derived neurotrophic factor (BDNF) is required for the enhancement of hippocampal neurogenesis following environmental enrichment. Eur $J$ Neurosci 24: 1850-1856, 2006.

ROSSI S, FURLAN R, DE CHIARA V, MUSELLA A, LO GIUDICE T, MATALUNI G, CAVASINNI F, CANTARELLA C, BERNARDI G, MUZIO L, MARTORANA A, MARTINO G, CENTONZE D: Exercise attenuates the clinical, synaptic and dendritic abnormalities of experimental autoimmune encephalomyelitis. Neurobiol Dis 36: 51-59, 2009.

SARCHIELLI P, GRECO L, STIPA A, FLORIDI A, GALLAI V: Brain-derived neurotrophic factor in patients with multiple sclerosis. J Neuroimmunol 132: 180-188, 2002.

SCHINDER AF, POO M: The neurotrophin hypothesis for synaptic plasticity. Trends Neurosci 23: 639-645, 2000.

SCHULZ KH, GOLD SM, WITTE J, BARTSCH K, LANG UE, HELLWEG R, REER R, BRAUMANN KM, HEESEN C: Impact of aerobic training on immune-endocrine parameters, neurotrophic factors, quality of life and coordinative function in multiple sclerosis. J Neurol Sci 225: 11-18, 2004.

SHARMA KR, KENT-BRAUN J, MYNHIER MA, WEINER MW, MILLER RG: Evidence of an abnormal intramuscular component of fatigue in multiple sclerosis. Muscle Nerve 18: 1403-1411, 1995.

SOUKUP T, ZACHAROVA G, SMERDU V: Fibre type composition of soleus and extensor digitorum longus muscles in normal female inbred Lewis rats. Acta Histochem 104: 399-405, 2002.

SOUKUP T, SMERDU V, ZACHAROVA G: Fiber type composition of unoperated rat soleus and extensor digitorum longus muscles after unilateral isotransplantation of a foreign muscle in long-term experiments. Physiol Res 58: 253-262, 2009. 
STUIFBERGEN AK: Physical activity and perceived health status in persons with multiple sclerosis. $J$ Neurosci Nurs 29: 238-243, 1997.

TAL C, BEHAR AJ: A demyelinating disease of the brain produced by injection of liver or liver proteolipid. $J$ Pathol Bacteriol 76: 483-490, 1958.

TAMAKI T, AKATSUKA A, TOKUNAGA M, ISHIGE K, UCHIYAMA S, SHIRAISHI T: Morphological and biochemical evidence of muscle hyperplasia following weight-lifting exercise in rats. Am J Physiol 273: C246C256, 1997.

TANAKA T, KARIYA Y, HOSHINO Y: Histochemical study on the changes in muscle fibers in relation to the effects of aging on recovery from muscular atrophy caused by disuse in rats. J Orthop Sci 9: 76-85, 2004.

THOMAS L, PATERSON PY, SMITHWICK B: Acute disseminated encephalomyelitis following immunization with homologous brain extracts; studies on the role of a circulating antibody in the production of the condition in dogs. J Exp Med 92: 133-152, 1950.

THOMPSON LV: Skeletal muscle adaptations with age, inactivity, and therapeutic exercise. J Orthop Sports Phys Ther 32: 44-57, 2002.

VANDENBARK AA, GILL T, OFFNER H: A myelin basic protein-specific T lymphocyte line that mediates experimental autoimmune encephalomyelitis. J Immunol 135: 223-228, 1985.

VOLTARELLI FA, GOBATTO CA, DE MELLO MA: Determination of anaerobic threshold in rats using the lactate minimum test. Braz J Med Biol Res 35: 1389-1394, 2002.

WENS I, DALGAS U, VANDENABEELE F, KREKELS M, GREVENDONK L, EIJNDE BO: Multiple sclerosis affects skeletal muscle characteristics. PLoS One 9: e108158, 2014.

WENS I, BROEKMANS T, HENDRIKS JJ, SAVELBERG HH, HESSELINK MK, EIJNDE BO: Effects of exercise intensity in experimental autoimmune encephalomyelitis. J Mult Scler 2: 2015.

WHITE LJ, DRESSENDORFER RH: Exercise and multiple sclerosis. Sports Medicine 34: 1077-1100, 2004.

WOOD S: Generalized Additive Models: An Introduction with R. Chapman \& Hall/CRC Press, London/Boca Raton, 2006.

YARASHESKI KE, LEMON PW, GILLOTEAUX J: Effect of heavy-resistance exercise training on muscle fiber composition in young rats. $J$ Appl Physiol 69: 434-437, 1990. 